\title{
Within-the-hour variability: levels and their probabilities
}

\author{
Stamatios S. Kouris $\left({ }^{1}\right)$, Kostas V. Polimeris $\left({ }^{1}\right)$, Vincenzo Romano $\left({ }^{2}\right)$, \\ Bruno Zolesi ( $\left(^{2}\right)$ and Ljiljana R. Cander $\left(^{3}\right)$ \\ $\left.{ }^{1}{ }^{1}\right)$ Division of Telecommunications, Department of Electrical Engineering, \\ Faculty of Engineering, Aristotle University of Thessaloniki, Greece \\ $\left.{ }^{2}\right)$ Istituto Nazionale di Geofisica e Vulcanologia, Roma, Italy \\ $\left(^{3}\right)$ Radio Communication Research Unit, Rutherford Appleton Laboratory, Chilton, Oxon, U.K.
}

\begin{abstract}
The study of foF 2 data measured every 5-min and of TEC measurements made every 10-min shows that the within-the-hour variability is different in the two parameters. Deciles of this variability for $f o F 2$ and for TEC are determined together with the probabilities of exceeding a given level of variability. Furthermore, considering hourly values, it is found that the variability in TEC is like an «intrinsic noise» throughout the day of the order of less than 5\% of the hourly value; but at sunrise and often at sunset large values take place. A seasonal dependence is evident. Besides, a within-the-hour variability in $f o F 2$ is always present with large values at sunrise or sunset depending on the season, and also during disturbed ionospheric conditions.
\end{abstract}

Key words ionospheric variability - radio wave propagation - space and satellite communications

\section{Introduction}

Perturbations of ionization density at the heights of the ionospheric $F$-region are permanent features. Therefore, meaningful longer term predictions can only have statistical character, i.e. specify probabilities (e.g., Rawer, 1957, 1993). Systematic studies on the day-today and hour-to-hour variability of the critical frequency of the $F$-region, foF 2 , and of the Total Electron Content, TEC, have shown that these perturbations, with negative and positive response, depend on local time, season and lo-

Mailing address: Prof. Stamatios S. Kouris, Division of Telecommunications, Department of Electrical Engineering, Faculty of Engineering, Aristotle University of Thessaloniki, Box 435, GR-54 124 Thessaloniki, Greece; email: kouris@vergina.eng.auth.gr cation (Kouris et al., 1998, 1999, 2002, 2006; Rawer et al., 2003; Fotiadis et al., 2004).

Regarding variations of $f o F 2$ in the time interval of an hour have been reported in different papers (Kouris et al., 2000; Fotiadis et al., 2001; Zolesi et al., 2001; Buresova and Laštovička, 2001) showing that a variation of $f o F 2$ around $12 \%$ (positive or negative) with respect to the hourly daily value measured at the standard-hour time is always present. This ionospheric variability «within-the-hour» or otherwise «ionospheric density noise» can be estimated from the variation of the relative deviations of 5-min daily foF 2 measurements with respect to the standard hour daily value of $f_{o F} 2$ measured at the corresponding standard hour. The diurnal variation of these 5-min relative deviations shows clearly that in certain hours and under particular circumstances, values of variability greater than $12 \%$ (in absolute value) of the corresponding hourly daily value can occur (Kouris et al., 2000; Buresova and Laštovička, 2001; Fotiadis et al., 2001). Moreover, variations in TEC in the time interval of an hour 
were reported as preliminary results in a previous paper (Polimeris et al., 2004) considering a limited number of data. It was then shown that the variability in TEC is rather negligible, except at sunrise and sunset where values up to $20 \%$ higher or lower of the corresponding daily standard hour value have been observed.

In the present work we investigate the variability within-the-hour in TEC at different European locations and, for comparison, in $f o F 2$ at the location of Rome. The statistical study of their variations in the interval of an hour allow us to determine bounds of the within-the-hour variability in $f o F 2$ and in TEC for all hours of the day in each month and season.

\section{Data and analysis}

We use foF2 5-min data measured at Rome $\left(41.9^{\circ} \mathrm{N}, 12.5^{\circ} \mathrm{E}\right)$ during the years $1997-2001$ and 10-min TEC data obtained from GPS measurements made mainly at Matera $\left(40^{\circ} \mathrm{N}, 16^{\circ} \mathrm{E}\right)$ during the years 1993 to 1999 , and at Hailsham $\left(50.9^{\circ} \mathrm{N}, 0.3^{\circ} \mathrm{E}\right)$ during 1998 to 2004 . We have also examined TEC data measured every 10min during August to December in 2002 to 2004 at the locations of Brussels $\left(50.8^{\circ} \mathrm{N}\right.$, $\left.4.3^{\circ} \mathrm{E}\right)$ and $\mathrm{Nicosia}\left(35.1^{\circ} \mathrm{N}, 33.2^{\circ} \mathrm{E}\right)$.

First we evaluate the 10 -min relative deviations of TEC from the measured every 10-min daily values in each interval time of an hour (half an hour before and after each standard hour) of each day/month/year/location using the expression

$$
\mathrm{d} T=\frac{T_{10}-T_{h}}{T_{h}}
$$

where $\mathrm{d} T$ is the 10 -min relative deviation, $T_{10}$ is the measured value of TEC every $10-\mathrm{min}$ and $T_{h}$ is the hourly measured value corresponding at the standard hour of the examined one hour interval time. A similar expression is used to calculate the variability within-the-hour of $f_{o F} 2$ (Fotiadis et al., 2001) that is

$$
\mathrm{d} f=\frac{f_{5}-f_{h}}{f_{h}}
$$

with $\mathrm{d} f$ the 5 -min relative deviation, $f_{5}$ the value of foF 2 measured every 5 -min and $f_{h}$ the corre- sponding hourly daily value of $f o F 2$ in the considered one hour interval.

In this analysis for each ionospheric parameter we deal with the relative deviations with respect to the standard hour value in the interval time of one hour, in order to remove the regular daily variation and to avoid any effect of the variability from hour-to-hour and from day-today on the obtained 5-min/10-min relative deviation, and so we may consider the totality of relative deviations as a whole.

Since we also compare the variability of within-the-hour with the variability from day-today, that is their deciles. Hourly TEC data and monthly median are also used from measurements made at the above referred locations and during the same periods. Then, the hourly relative deviation is estimated using the expression

$$
\mathrm{d} T=\frac{T_{h}-T_{m}}{T_{m}}
$$

where $\mathrm{d} T$ is now the hourly relative deviation, $T_{h}$ is the hourly measured value and $T_{m}$ is the corresponding monthly median value. Evident$1 y$, the variation of $\mathrm{d} T$ from day-to-day describes the day-to-day variability of TEC at the given hour. Of course a similar procedure is used in the case of $f_{o F}$.

\section{Results and discussion}

The counted relative deviations are first studied month by month and then by season to investigate any annual or seasonal dependence. To inquire whether deviations as high as $20 \%$ of the corresponding standard hour value are related to magnetic disturbances, the calculated $\mathrm{d} f$ and $\mathrm{d} T$ values have been studied day by day.

Variability in TEC - The analysis shows that the within-the-hour variability in TEC has a different behavior from that in $f o F 2$. It is found that a variation in TEC of $10 \%$ with respect to the standard hour value has a probability much less than $10 \%$ if not zero, contrary to what is found for $f o F 2$. Indeed, only an «intrinsic» variability of the order of less than $5 \%$ is found, comparative to the one found for all other ionospheric characteristics, e.g. foE (Kouris and Fotiadis, 2002). Table I reports probabilities 
Table I. Probability the level of variability within-the-hour in TEC exceeds the value of 0.05 (A), 0.10 (B), 0.15 (C), 0.20 (D). Data measured at Hailsham during $2002\left(80<R_{12}<120\right)$.

\begin{tabular}{|c|c|c|c|c|c|c|c|c|c|c|c|c|c|c|c|c|c|c|c|c|c|c|c|c|}
\hline $\mathbf{A}$ & & & & & 4 & 5 & & 7 & & & 10 & & 12 & 13 & 14 & 15 & 6 & 17 & 18 & 19 & 20 & 21 & 22 & \\
\hline & & & & 6 & 15 & & & & & & & & & & & & & & & & & & & \\
\hline & & & 16 & 1 & 2 & & & & & & & & & & & & & & & & & & & \\
\hline & & & & & 0 & & & & & & & & & & & & & & & & & & & \\
\hline & & & & & 3 & & & & & & & & & & & & & & & & & & & \\
\hline & & & 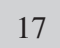 & & 63 & & 33 & & & & & & & & & & & & & & & & & \\
\hline $\mathrm{J}$ & & & 14 & & 5 & & 9 & & 2 & & & & & & & & & & & & & & & \\
\hline I & & & & & 6 & & & & & & & & & & & & & & & & & & & \\
\hline$A$ & & & 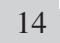 & & 3 & & & & & & & & & & & & & & & & & & & \\
\hline S & 5 & & 21 & & 2 & 1 & 9 & & 4 & & & & & & & & & & & & & & & \\
\hline & & & & & 3 & & & & & & & & & & & & & & & & & & & \\
\hline & & & 27 & 2 & 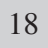 & 0 & 55 & & $\mathrm{c}$ & 72 & & & & 8 & & 5 & & & & & & & & \\
\hline $\mathrm{D}$ & & & 16 & & 19 & 3 & 21 & & 1 & 89 & & & & 8 & & 43 & & & & & & & & \\
\hline & & 1 & 2 & 3 & 4 & 5 & & & & & & & & & & & & & & & & & & \\
\hline & & & 0 & & 07 & & & & 03 & 48 & & & & & & & & & & & & & & \\
\hline & c & & 0.7 & 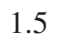 & 0 & & 30 & & 6 & 3 & & & & & & & & & & & & & & \\
\hline & & 0 & 0 & & 2.7 & & 9 & & & & & & & & & & & & & & & & & \\
\hline & & 4.1 & 3.4 & 5.5 & 14 & & 28 & & 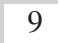 & & & & & & & 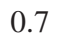 & & & & & & & & \\
\hline & 5 & .3 & 4 & 4 & 23 & 15 & 4.7 & 3.3 & 1.3 & & ( & & & & & & & & & & & 7 & & \\
\hline & & ( & ( & 0 & 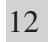 & & 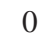 & & & & & & & & & & & & & & & & & \\
\hline $\mathbf{J}$ & & 0 & 2 & 0 & 21 & 6 & & & & & & & & & & & & & & & & & & \\
\hline $\mathrm{A}$ & & & 1 & 3.2 & 16 & 2 & 20 & 5.2 & & 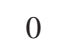 & & & & & & & & & & & & & & \\
\hline & & & 2 & 2 & 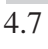 & & & & & & & & & & & & & & & & & & & \\
\hline 0 & & & & 73 & 6.7 & 12 & 7 & 5 & 2 & 1 & & & & & & & & & & 8 & & & & \\
\hline$N_{-1}$ & & & & & 4.1 & 3. & 29 & & 6 & & & & & & & 1 & & & & & .6 & & & \\
\hline L & & & & & - & & 5.2 & & 0 & 51 & & & & & & & & & & & & & & \\
\hline & & & 0 & 3 & 4 & - & & & & & & & & & & & & & & & & & & \\
\hline & & & & & & & & & & & & & & & & & & & & & & & & \\
\hline & & & ( & & 0 & 0 & & & & & & & & & & & & & & & & & & \\
\hline & & 0 & 0 & & 1.3 & & & & 4.7 & & & & & & & & & & & 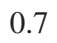 & & & & \\
\hline A & & 8 & 2.8 & 3.4 & 6.2 & 15 & 10 & 3.4 & 0 & 7 & & & & & & & & & & & & .1 & .7 & \\
\hline & & & 2 & 2 & & 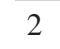 & & 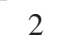 & & ช & & & & & & & & & & & & & & \\
\hline $\mathbf{J}$ & 0 & 0 & & 0 & & 0 & 0 & & & & & & & & & & & & & & & & & \\
\hline $\mathrm{J}$ & 0 & 0 & 0 & 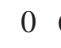 & & & & & & & & & & & & & & & & & & & & \\
\hline$A$ & & & 0 & & 5.8 & & & 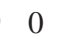 & & & & & & & & & & & & & & & & \\
\hline $\mathrm{S}$ & & 0 & 0 & 0 & 0 & 26 & & & & & & & & & & & & & & & & & & \\
\hline $\mathrm{O}$ & & 2. & 4 & & 1.3 & 5.3 & $4 J$ & 31 & 8.7 & & & & & & & 0.1 & & & .7 & 13 & 2.7 & 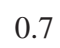 & & \\
\hline 1 & & 0.7 & 0 & & & & 19 & & 46 & & & & & & & 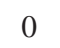 & & & & & 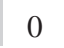 & & & \\
\hline & 0 & 0 & 0 & & & 20 & 1.9 & 5 & 62 & 4 & & & & & & 0 & & & 23 & 8 & 3.4 & - & & \\
\hline
\end{tabular}


Table I (continued).

\begin{tabular}{l|llllllllllllllllllllllll}
\hline $\mathbf{D}$ & 0 & 1 & 2 & 3 & 4 & 5 & 6 & 7 & 8 & 9 & 10 & 11 & 12 & 13 & 14 & 15 & 16 & 17 & 18 & 19 & 20 & 21 & 22 & 23 \\
\hline
\end{tabular} \begin{tabular}{l|llllllllllllllllllllllll}
\hline $\mathrm{J}$ & 0 & 0 & 0 & 0 & 0 & 0 & 0 & 43 & 55 & 6 & 0 & 0 & 0 & 0 & 0 & 0 & 0 & 2.7 & 6 & 0.7 & 0 & 0.7 & 2.7 & 0
\end{tabular} $\mathrm{F} \quad \begin{array}{llllllllllllllllllllllll}0 & 0 & 0 & 0 & 0 & 0 & 12 & 52 & 24 & 0.7 & 0 & 0 & 0 & 0 & 0 & 0 & 0 & 0 & 0 & 0 & 0 & 0 & 0 & 0\end{array}$ M $\quad \begin{array}{llllllllllllllllllllllll}0 & 0 & 0 & 0 & 0.7 & 4.7 & 29 & 17 & 2 & 0.7 & 0 & 0 & 0 & 0 & 0 & 0 & 0 & 0 & 0 & 0 & 0.7 & 0 & 0 & 0\end{array}$ A $\quad \begin{array}{llllllllllllllllllllllll}1.7 & 1.4 & 2.8 & 2.8 & 3.4 & 6.9 & 1.4 & 0 & 0 & 0 & 0.7 & 0 & 0 & 0 & 0 & 0 & 0 & 0 & 0 & 0 & 2.1 & 0.7 & 0 & 2.8\end{array}$

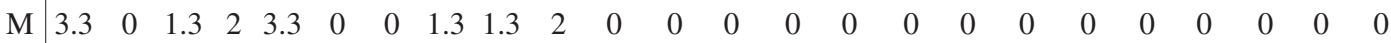

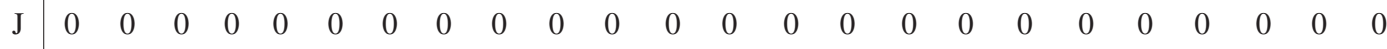

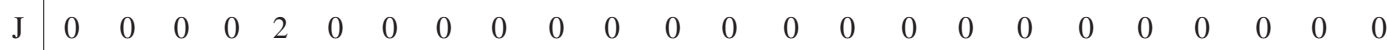

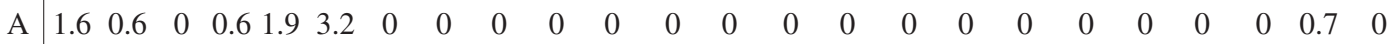
$\mathrm{S} \quad 0 \begin{array}{llllllllllllllllllllllll}0 & 0 & 0 & 0 & 13 & 8.7 & 1.3 & 0 & 0 & 0 & 0 & 0 & 0 & 0 & 0 & 0 & 0 & 0 & 0.7 & 1.3 & 0.7 & 0 & 0.7\end{array}$

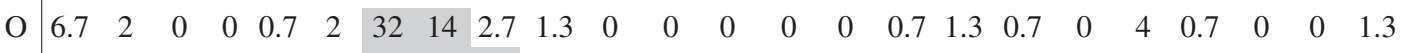
$\mathrm{N} \quad \begin{array}{llllllllllllllllllllllll}3.4 & 0.7 & 0 & 0 & 0.7 & 0 & 12 & 56 & 19 & 0 & 0 & 0 & 0 & 0 & 0 & 0 & 0 & 2.1 & 9.7 & 0.7 & 0 & 0.7 & 2.1 & 5.5\end{array}$ $\begin{array}{lllllllllllllllllllllllll}\mathrm{D} & 0 & 0 & 0 & 0 & 0 & 1.9 & 0 & 40 & 43 & 3.3 & 0 & 0 & 0 & 0 & 0 & 0 & 2.1 & 6.9 & 5.5 & 0 & 4.1 & 4.1 & 0.7 & 0\end{array}$

Table II. Probability the variability in TEC may exceeds the bounds 0.05 (A), 0.10 (B) or 0.20 (C). Data from Matera $1996\left(R_{12}<10.5\right)$.

\begin{tabular}{|c|c|c|c|c|c|c|c|c|c|c|c|c|c|c|c|c|c|c|c|c|c|c|c|c|}
\hline A & & & 2 & 3 & 4 & 5 & & & 8 & 9 & 10 & 11 & 12 & 13 & 14 & 5 & 16 & 17 & 18 & 9 & 20 & 1 & 2 & \\
\hline & & & & & & & & & & & & & & & & & & & & & & & & \\
\hline $\mathrm{F}_{2}$ & & & & & & & & & & & 1 & & & & & & & & & & & & & \\
\hline 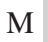 & & & & & & & & & & & 12 & & & & & 4 & & & & & & & & \\
\hline A & & & & & & & & & & & & & & & & 8 & & & & & & & & \\
\hline & & & & & & & & & & & & & & & & & & & & & & & & \\
\hline J & & 4 & & & 9 & 7 & & 1 & 3 & 7.4 & 3 & 7 & & 3 & & 4.1 & 3 & & & & & & & \\
\hline J & & 2 & & & & & & 19 & & ) & 9. & 8. & 11 & & 9.3 & 4.3 & & & & & & & & \\
\hline A & & & & & & & & 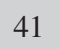 & & & & & & & & & & & & & & & & \\
\hline S & 50 & & & & & & 4 & 4 & & 9 & 1 & 16 & 1 & & 8.7 & 11 & & & & & & & & \\
\hline $\mathrm{O}$ & & 26 & & & & 9 & & 60 & 2 & 2 & 19 & 6. & 2 & & 18 & 26 & 40 & & & 1 & & & & \\
\hline V & & & & & & & & & & & & & & & & & & & & & & & & \\
\hline D & 10 & 15 & & 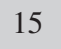 & 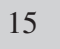 & 32 & อ? & 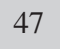 & & 25 & & & & & 23 & 49 & & & & & & & & \\
\hline $\mathbf{D}$ & & 1 & 2 & 2 & 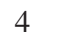 & 5 & & & & 0 & & & & & & & & & & & & & & \\
\hline J & & & & & & & & & S & & & & & & & & & & & & & & & \\
\hline 1 & & & 5 & & 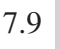 & 21 & 58 & & & & 0. & & 0 & & & J & & & & & & & & \\
\hline M & 1 & 7 & 12 & 5.2 & 0 & 50 & 2 & 7 & 2 & 2 & 0 & 0.7 & 1.3 & 0 & 2.6 & 1.3 & 1.9 & 9 & 11 & .4 & 0 & & & \\
\hline $\mathrm{A}$ & & & & 6 & 41 & 3 & 11 & 4 & & 0.7 & & & 0 & & 0.7 & 0.7 & 0 & & & 9 & 3 & 4 & & \\
\hline $\mathbf{M}$ & & & & 13 & 6 & 33 & & 0 & & & ( & & & & 0 & & & & & 2.7 & 8.8 & & & \\
\hline 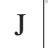 & 5.6 & 13 & & 38 & 5 & 30 & 4.9 & 0 & & 1 & 0 & & & & 0 & 0 & & & & 4 & (6) & & & \\
\hline $\mathrm{J}$ & & 9.6 & & 20 & 6 & 2 & 11 & 5.8 & 3.3 & 8 & & & 2.9 & 1.4 & 1.4 & 0 & U & & & 7.9 & y & & & \\
\hline A & & 29 & & 18 & 5 & & & 18 & 6 & 5.3 & 3 & & & & ( & & & & & .5 & 8 & ) & & \\
\hline . & 32 & 4 & 15 & 6 & 28 & 0 & 12 & 7.7 & 7.6 & 0 & & 6. & & & 6 & 4.3 & & & & 16 & & & & \\
\hline $\mathrm{O}$ & & & 1 & 05 , & 76 & 63 & 4 & 15 & 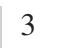 & 6 & 3 & 1 & 5.7 & 5.7 & 5.9 & 8 & 20 & 36 & 25 & 15 & 18 & 30 & 52 & \\
\hline
\end{tabular}


Table II (continued).

\begin{tabular}{c|cccccccccccccccccccccccc}
\hline \hline $\mathbf{B}$ & 0 & 1 & 2 & 3 & 4 & 5 & 6 & 7 & 8 & 9 & 10 & 11 & 12 & 13 & 14 & 15 & 16 & 17 & 18 & 19 & 20 & 21 & 22 & 23 \\
\hline $\mathrm{N}$ & 0 & 1.3 & 2.5 & 16 & 11 & 21 & 60 & 10 & 7.5 & 1.3 & 2.5 & 0 & 3.8 & 6.3 & 9.1 & 10 & 11 & 1.5 & 1.5 & 0 & 1.5 & 0 & 0 & 0 \\
$\mathrm{D}$ & 3.3 & 4.7 & 4 & 3.3 & 2.7 & 10 & 41 & 11 & 15 & 3.3 & 0 & 2 & 0 & 0.7 & 3.3 & 15 & 13 & 0.7 & 0.7 & 0.7 & 0.7 & 1.3 & 0.7 & 0.7 \\
\hline $\mathbf{C}$ & 0 & 1 & 2 & 3 & 4 & 5 & 6 & 7 & 8 & 9 & 10 & 11 & 12 & 13 & 14 & 15 & 16 & 17 & 18 & 19 & 20 & 21 & 22 & 23 \\
\hline $\mathrm{J}$ & 5.2 & 1.3 & 0.7 & 0 & 0 & 1.9 & 19 & 1.3 & 0.6 & 0.7 & 0 & 0 & 0 & 0 & 0 & 0 & 0 & 0 & 0 & 3.2 & 0.6 & 0 & 0 & 0 \\
$\mathrm{~F}$ & 0 & 0 & 0.7 & 0 & 1.4 & 4.3 & 14 & 0 & 0 & 0 & 0 & 0 & 0 & 0 & 0 & 0 & 0 & 0 & 0 & 0 & 0 & 0 & 0 & 0 \\
$\mathrm{M}$ & 5.4 & 0 & 1.9 & 1.3 & 0 & 16 & 0.6 & 0 & 0 & 0 & 0 & 0 & 0 & 0 & 0 & 0 & 0 & 0 & 0 & 0 & 0 & 0 & 0 & 0 \\
$\mathrm{~A}$ & 0 & 0.7 & 0 & 0 & 15 & 5.3 & 0 & 0 & 0 & 0 & 0 & 0 & 0 & 0 & 0 & 0 & 0 & 0 & 0 & 0 & 0.7 & 0 & 1.4 & 0.7 \\
$\mathrm{M}$ & 1.7 & 0 & 0 & 4.7 & 18 & 0.7 & 0 & 0 & 0 & 0 & 0 & 0 & 0 & 0 & 0 & 0 & 0 & 0 & 0 & 0 & 0 & 0.7 & 0 & 0 \\
$\mathrm{~J}$ & 3.7 & 2.1 & 0.7 & 17 & 16 & 2.1 & 0 & 0 & 0 & 0 & 0 & 0 & 0 & 0 & 0 & 0 & 0 & 0 & 0 & 0 & 1.3 & 0 & 0 & 0 \\
$\mathrm{~J}$ & 9.5 & 3.5 & 1.8 & 6.7 & 24 & 1 & 7.9 & 0 & 0 & 6 & 0 & 0 & 0 & 0 & 0 & 0 & 0 & 0 & 0 & 0 & 3.6 & 0 & 0 & 1.5 \\
$\mathrm{~A}$ & 12 & 7.9 & 11 & 6.8 & 26 & 5.3 & 6 & 6 & 0.7 & 1.3 & 0 & 0 & 0 & 0 & 0 & 0 & 0 & 0 & 0 & 0.7 & 1.4 & 0 & 0 & 1.4 \\
$\mathrm{~S}$ & 7.1 & 0 & 5.2 & 1 & 7 & 13 & 1 & 0 & 0 & 0 & 3.6 & 3.5 & 0.9 & 0 & 0 & 3.5 & 1.8 & 0.9 & 0.9 & 2.7 & 0 & 0.9 & 2.9 & 0 \\
$\mathrm{O}$ & 4.8 & 1 & 0 & 1.9 & 3.8 & 23 & 2.9 & 0 & 0 & 1 & 0 & 0 & 1 & 1.9 & 2 & 2 & 4 & 14 & 12 & 5.9 & 2.5 & 18 & 42 & 21 \\
$\mathrm{~N}$ & 0 & 0 & 0 & 7.5 & 5 & 10 & 13 & 0 & 1.3 & 0 & 0 & 0 & 0 & 1.3 & 6.5 & 4.4 & 0 & 0 & 0 & 0 & 0 & 0 & 0 & 0 \\
$\mathrm{D}$ & 0 & 1.3 & 0.7 & 0 & 0 & 0 & 8 & 0.7 & 0.7 & 0 & 0 & 2 & 0 & 0 & 0.7 & 1.3 & 0 & 0 & 0 & 0 & 0 & 0 & 0 & 0 \\
\hline
\end{tabular}

Table III. Probability the within-the-hour variability in TEC be greater than 0.10 (A, C) or 0.20 (B, D). Data measured in 1999; Matera (A, B) and Hailsham (C, D). High solar activity $\left(80<R_{12}<120\right)$.

\begin{tabular}{c|cccccccccccccccccccccccc}
\hline \hline $\mathbf{A}$ & 0 & 1 & 2 & 3 & 4 & 5 & 6 & 7 & 8 & 9 & 10 & 11 & 12 & 13 & 14 & 15 & 16 & 17 & 18 & 19 & 20 & 21 & 22 & 23 \\
\hline $\mathrm{J}$ & 10 & 7.1 & 8.4 & 5.2 & 3.9 & 4.5 & 76 & 39 & 12 & 6 & 0 & 0 & 0.6 & 0.6 & 3.9 & 17 & 31 & 21 & 5.2 & 1.9 & 3.2 & 0 & 0.6 & 0 \\
$\mathrm{~F}$ & 6.3 & 2.4 & 0 & 0 & 0 & 26 & 82 & 15 & 5.7 & 2.1 & 0 & 0 & 0 & 0 & 0 & 4.3 & 20 & 29 & 8.6 & 8.6 & 0.7 & 0 & 0.7 & 1.4 \\
$\mathrm{M}$ & 0 & 0.7 & 0 & 2.2 & 3 & 69 & 40 & 19 & 7.2 & 4.7 & 0 & 0 & 0.6 & 1.4 & 0 & 0.7 & 4.5 & 12 & 28 & 14 & 2.6 & 0.6 & 0 & 3.2 \\
$\mathrm{~A}$ & 0 & 0.9 & 0.9 & 6.1 & 40 & 39 & 18 & 9 & 6.2 & 0 & 0 & 0.7 & 0 & 0 & 0 & 0 & 0 & 0 & 12 & 13 & 4.4 & 2.2 & 0 & 0 \\
$\mathrm{M}$ & 0 & 0 & 0.7 & 7.1 & 50 & 11 & 2.7 & 0 & 0 & 0.7 & 0 & 0 & 0 & 0 & 0 & 0 & 0 & 0 & 0 & 3.3 & 1.4 & 0 & 0 & 0 \\
$\mathrm{~J}$ & 1.7 & 0 & 0 & 16 & 30 & 2.1 & 0 & 0 & 0 & 0 & 0 & 0 & 0 & 0.7 & 0 & 0 & 0 & 0 & 0 & 0 & 0 & 0.7 & 0 & 0 \\
$\mathrm{~J}$ & 0 & 0.7 & 2 & 4 & 25 & 3.3 & 0 & 0 & 0 & 0 & 0 & 0 & 0 & 0 & 0 & 0 & 0 & 0 & 0 & 0 & 0.7 & 2 & 2 & 0 \\
$\mathrm{~A}$ & 1.7 & 0.7 & 0.7 & 0.7 & 40 & 26 & 3.4 & 0 & 0.7 & 0 & 0 & 0 & 0 & 0 & 0 & 0.7 & 0 & 0 & 0 & 4.8 & 4.1 & 2.1 & 0 & 0 \\
$\mathrm{~S}$ & 0 & 0.7 & 1.4 & 0.7 & 23 & 52 & 12 & 6.5 & 4.3 & 0.7 & 0.7 & 0 & 0 & 0 & 0.7 & 0.7 & 0 & 5 & 8.6 & 2.1 & 2.9 & 0 & 0 & 0 \\
$\mathrm{O}$ & 0 & 0.8 & 0 & 0 & 1.6 & 73 & 57 & 18 & 1.6 & 0.8 & 0 & 0 & 0 & 0 & 0 & 3.2 & 19 & 26 & 10 & 2.4 & 2.3 & 0 & 0 & 2.3 \\
$\mathrm{~N}$ & 0 & 0 & 0.7 & 2.1 & 0 & 41 & 81 & 43 & 11 & 0 & 0.7 & 0 & 0 & 0 & 1.3 & 17 & 38 & 24 & 8 & 2.7 & 0 & 0 & 0 & 0.7 \\
$\mathrm{D}$ & 1.7 & 1.3 & 0.7 & 1.3 & 0 & 15 & 86 & 39 & 21 & 4.7 & 2.1 & 0 & 0 & 0 & 2 & 22 & 42 & 19 & 6.7 & 2.7 & 2 & 0 & 0 & 0 \\
\hline $\mathbf{B}$ & 0 & 1 & 2 & 3 & 4 & 5 & 6 & 7 & 8 & 9 & 10 & 11 & 12 & 13 & 14 & 15 & 16 & 17 & 18 & 19 & 20 & 21 & 22 & 23 \\
\hline $\mathrm{J}$ & 0 & 2.6 & 1.3 & 0 & 0 & 0.6 & 34 & 3.2 & 0 & 0 & 0 & 0 & 0 & 0 & 0 & 0 & 0 & 0.6 & 0 & 0 & 0 & 0 & 0 & 0 \\
$\mathrm{~F}$ & 0 & 0.8 & 0 & 0 & 0 & 11 & 39 & 0.8 & 0 & 0 & 0 & 0 & 0 & 0 & 0 & 0 & 0.7 & 0 & 0 & 0 & 0 & 0 & 0.7 & 0 \\
$\mathrm{M}$ & 0 & 0 & 0 & 0 & 0 & 28 & 7.4 & 2.1 & 0 & 0 & 0 & 0 & 0 & 0 & 0 & 0 & 0 & 1.3 & 0.6 & 1.3 & 0 & 0 & 0 & 0 \\
$\mathrm{~A}$ & 0 & 0 & 0 & 0 & 11 & 6.1 & 0 & 0 & 0 & 0 & 0 & 0 & 0 & 0 & 0 & 0 & 0 & 0 & 0 & 0 & 0 & 0 & 0 & 0 \\
$\mathrm{M}$ & 0 & 0 & 0 & 0 & 7.1 & 0 & 0 & 0 & 0 & 0 & 0 & 0 & 0 & 0 & 0 & 0 & 0 & 0 & 0 & 0 & 0 & 0 & 0 & 0 \\
$\mathrm{~J}$ & 0 & 0 & 0 & 0.7 & 0.7 & 0 & 0 & 0 & 0 & 0 & 0 & 0 & 0 & 0 & 0 & 0 & 0 & 0 & 0 & 0 & 0 & 0 & 0 & 0 \\
\hline
\end{tabular}


Table III (continued).

\begin{tabular}{|c|c|c|c|c|c|c|c|c|c|c|c|c|c|c|c|c|c|c|c|c|c|c|c|c|}
\hline & 0 & 1 & 2 & 3 & 4 & 5 & 6 & 7 & 8 & 9 & 10 & 11 & 12 & 13 & 14 & 15 & 16 & 7 & 8 & 9 & 0 & 21 & 22 & 23 \\
\hline & 0 & 0 & 0 & 0 & & 0 & 0 & 0 & & & & & & & & & & & & & & & & \\
\hline & 0 & 0 & $\Omega_{0}$ & 0 & & & 0 & & & & & & & & & & & & & & & & & 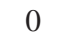 \\
\hline$S$ & 0 & 0 & 0 & 0 & 3 & 9 & 0 & 0 & ( & & & & & & & & & & & & & & & 0 \\
\hline & 0 & 0 & 0 & 0 & 0 & 32 & 8.8 & 0 & 0 & & 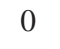 & & & & & ) & 0.8 & & & ) & 0.8 & U & & 0.8 \\
\hline & 0 & 0 & 0 & 0 & 0 & 20 & 38 & 2.1 & 0 & & & & & & & 0 & & & & & & & & 0 \\
\hline & 0 & 0 & 0.7 & 0 & 0 & 2 & 0 & 2 & 0 & 0 & & & & & 0 & 13 & 2 & & & 0 & & 0 & & 0 \\
\hline & 0 & 1 & 2 & 3 & 4 & 5 & 6 & 7 & 8 & 9 & 1 & 1 & & 13 & 14 & 15 & 16 & 17 & 18 & 19 & 20 & 21 & 22 & 23 \\
\hline 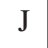 & 3.3 & 0 & 2.7 & 0 & 3 & 0.7 & 0 & 8 & 7 & 3 & 0 & 0 & & 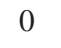 & O & 3 & 15 & 34 & 37 & 12 & 9 & 1.4 & 0 & 3. \\
\hline $\mathrm{F}$ & 0 & 0 & 0 & 1.6 & 1.6 & 2.4 & 26 & 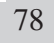 & 52 & 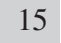 & 3.2 & 0 & ( & 0 & 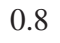 & 0.0 & 6 & & 3 & 27 & 8 & 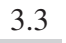 & & 0.8 \\
\hline 4 & 0 & 0.7 & 0.7 & 0 & 2.8 & 5 & 70 & 47 & 17 & 12 & 2 & 0 & & & & 0 & 0.7 & & & 18 & & 8 & & 2 \\
\hline & 0 & 0 & 0.7 & 3 & 9 & 51 & 32 & 13 & 1.5 & 1.5 & 0 & & & & & 0 & 1.5 & & 0 & 7 & 5.9 & 12 & 3.1 & 2.2 \\
\hline & 0 & 0 & 0 & 2 & 2 & 17 & 2.7 & 0.7 & 0 & & & & & & & & & & & 0.7 & 0. & 0.7 & & 0 \\
\hline & 0 & 0 & 0 & 0 & 10 & 6.2 & 0 & 0 & 0 & & 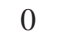 & & & & & & & & & & & 0 & & 0 \\
\hline $\mathrm{J}$ & 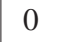 & & 2 & 1.3 & 13 & + & 0 & 0 & ( & 0 & & & & & & & & & & & & & & 2.7 \\
\hline A & 4 & & 6.2 & 5.5 & 20 & 43 & 13 & 9.3 & 2 & 0 & ( & ( & & & & & & & & J & & 8.3 & & 4.1 \\
\hline S & 4 & 4.4 & 2.2 & 5.22 & 2.2 & 39 & 50 & 19 & 3 & 5.2 & 0. & 0 & & U & 0.7 & 0 & 2.2 & & 0.8 & 7.7 & 16 & 11 & 3 & 0 \\
\hline $\mathrm{O}$ & 0 & 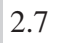 & 7 & 4.7 & 4.7 & 1 & 7 & 5 & & 1 & & & & & 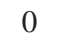 & & 2 & & & 3 & & 3 & & 0 \\
\hline $\mathrm{N}$ & 6 & 0 & - & 0 & 2.1 & 0 & 28 & 88 & 6 & $J$ & 1 & ( & & ( & 0 & 4.8 & 21 & & 4 & 23 & & 0.7 & & 0 \\
\hline $\mathrm{D}$ & 0 & 5.3 & 0.7 & 0 & 0 & 0 & 0.7 & 62 & 78 & 43 & 2 & & & & 0.7 & 1 & 29 & & 8 & 8 & 1.3 & 2 & 4 & 9.7 \\
\hline & 0 & 1 & 2 & 3 & 4 & 5 & 6 & 7 & 8 & 9 & 1 & 1 & 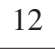 & 13 & 1 & 15 & 16 & 17 & 18 & 19 & 20 & 2 & 22 & 23 \\
\hline & 0 & 0 & 0 & 0 & 0 & 0 & 0 & 52 & 80 & & 0 & & & & & & & & & 7 & & & & 1.4 \\
\hline $\mathrm{F}$ & 0 & 0 & 0 & 0 & 0 & 0 & 12 & 36 & 4 & 0 & ( & & & & & 0 & & & 5 & 0.8 & & & & 0 \\
\hline & 0 & 0 & 0 & 0 & . & 2 & 31 & 6 & 2 & 2 & & & & & & & & & & 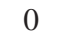 & & 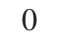 & & 0.7 \\
\hline & 0 & 0 & 0 & 0 & 0 & 16 & 2.2 & 1.5 & 0 & & & & & & & & & & 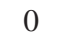 & 0 & ) & 0.7 & & 0 \\
\hline 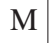 & 0 & 0 & 0 & 0 & 0.7 & 0.7 & 0 & 0 & 0 & & ( & & & & & & 0 & & & 0 & & 0 & & 0 \\
\hline $\mathrm{J}$ & 0 & 0 & o & 0 & . & 0 & 0 & 0 & ( & & & & & & & & & & & & & & & 0 \\
\hline$I_{1}$ & 0 & 0 & & 0 & & 0 & 0 & 0 & 0 & & 0 & & & & & & & & & 0 & & & & 0 \\
\hline A & 0 & 0 & 1.4 & 0 & 5.4 & 1 & 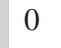 & 0 & 8 & ( & ( & & & & & & 0 & & 0 & 0 & J & 0 & ) & 1.4 \\
\hline$S$ & & 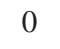 & 0 & 0 & 0 & 13 & & 1.5 & 0 & ( & ( & & & & & & 0 & & 0 & 0.8 & 1 & 0 & & 0 \\
\hline $\mathrm{O}$ & 3.3 & 0 & 0 & 1.3 & 0 & 4 & 33 & 15 & 1.3 & 0 & 0 & 0 & & & 0 & & 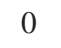 & ( & 0.7 & 2.7 & 3.3 & 0 & & 0 \\
\hline $\mathrm{N}$ & 1.7 & 0 & 0 & 0 & 0 & 0 & 9.7 & 48 & 13 & & 0 & & & & c & & 0.7 & & $3 .{ }^{\circ}$ & 0 & & 0 & 0 & 0 \\
\hline D & 0 & 2 & 0 & 0 & 0 & 0 & 0 & 32 & 35 & 0.7 & 0 & ( & ( & & 0 & 0 & 0.7 & 1.3 & 2.7 & 0 & & 0 & 2 & \\
\hline
\end{tabular}

that the level of variability in TEC exceeds given bounds during 2002, a year of high solar activity $\left(80<R_{12}<120\right)$ at Hailsham while table II reports probabilities from data measured at Matera during 1996, a year of low solar activi- ty $\left(R_{12}<10.5\right)$. It can be seen that the variability exceeds the limit of $5 \%$ mostly during the night-time hours (table I) and also under disturbed ionospheric conditions (table II). Thus, we may deduce that the variability in TEC with- 
in the interval of an hour, if any, is very low except around the sunrise hours and often around sunset too. The latter usually occurs in winter and equinoxes as can be seen from table III, where the probabilities that the within-the-hour variability in TEC be greater than given levels at two locations of different latitude and during 1999 a year of rather high solar activity $\left(80<R_{12}<115\right)$ are shown. At sunrise and sunset the probability of value of variability withinthe-hour greater than $10 \%$ and less than $30 \%$ is very high reaching values up to $60 \%-80 \%$. These high values of variability are observed during winter months and even during local disturbed conditions (tables I and II).

Figure 1 illustrates from measurements made at Hailsham the probability level that the variability within-the-hour be greater in ab-
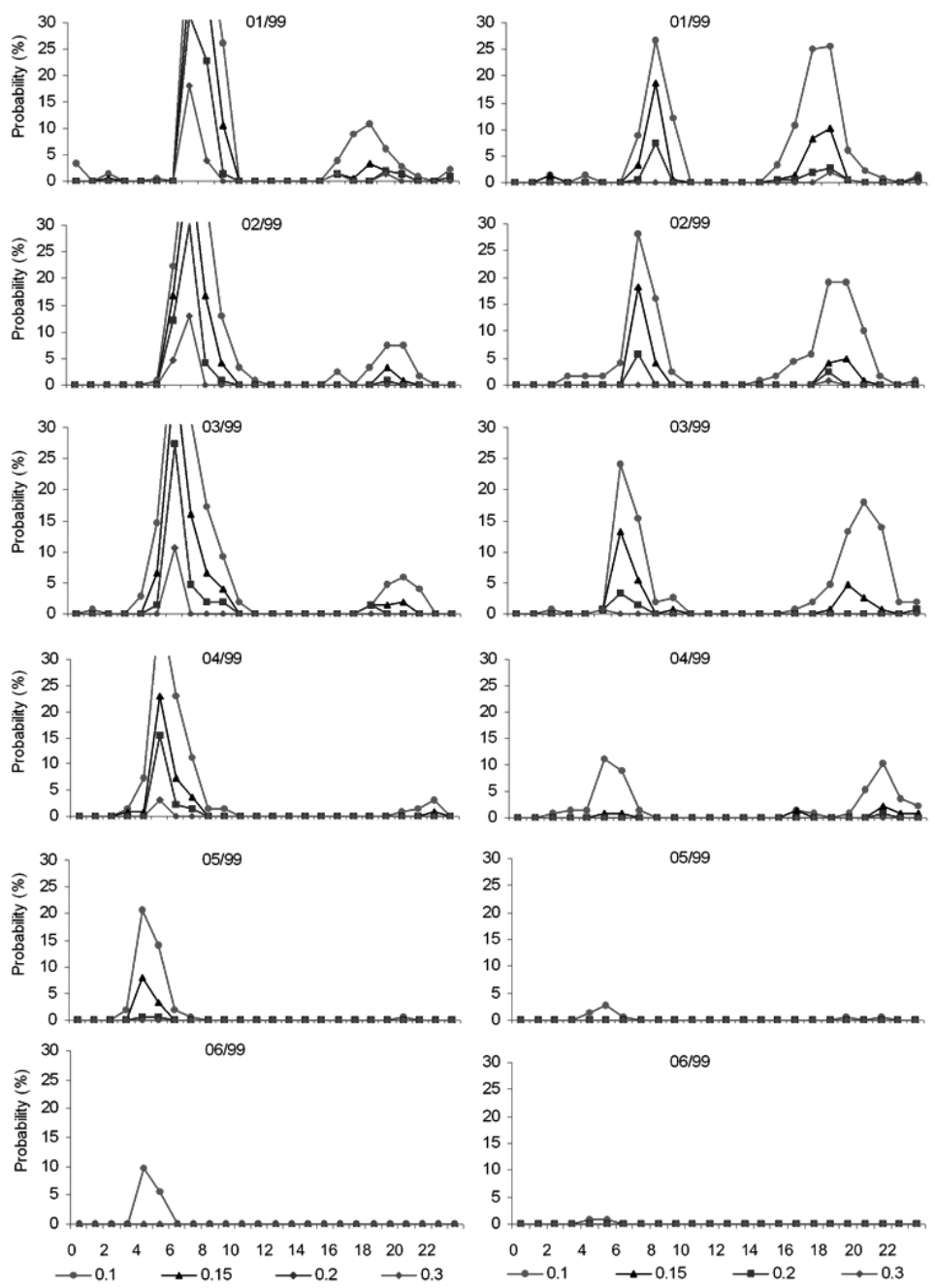

Fig. 1. Probability the variability within-the-hour in TEC, positive (right) or negative (left), be greater than $0.10,0.15,0.20$ or 0.30 , in absolute value. Data from Hailsham. 

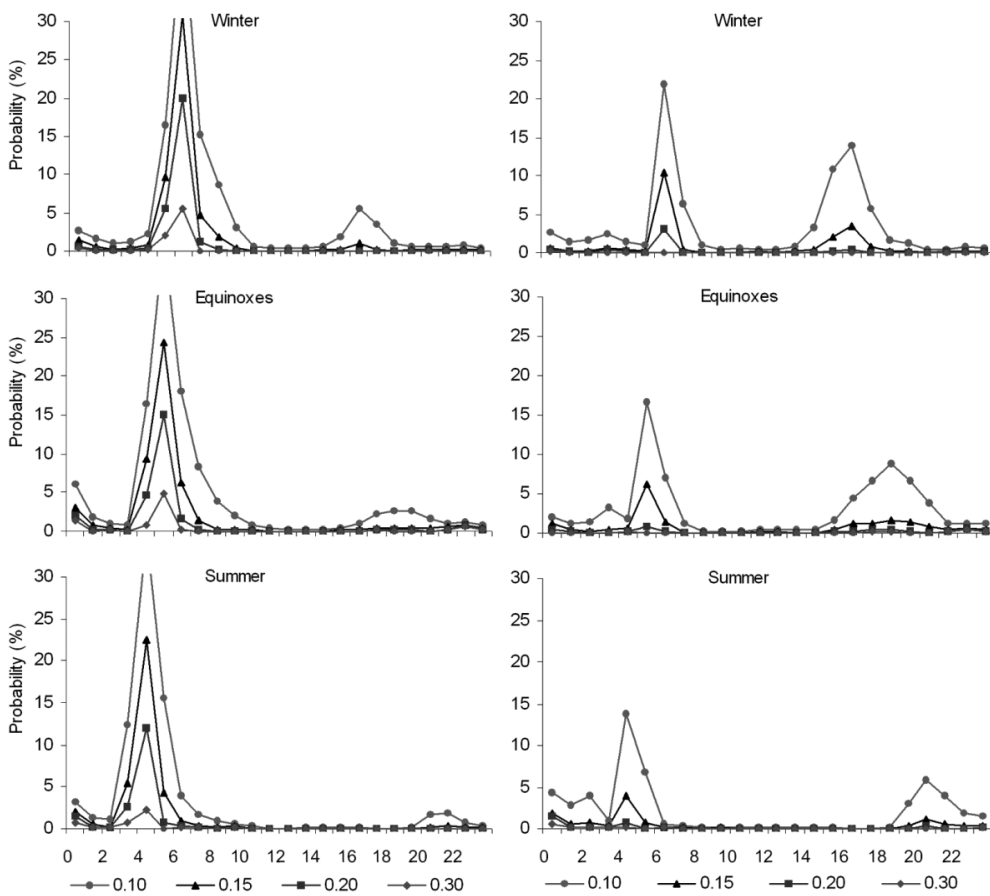

Fig. 2. Probability the variability within-the-hour in TEC, positive (right) or negative (left), be greater than $0.10,0.15,0.20$ or 0.30 , in absolute value. Data measured at Matera 1993-1999.

solute value than 0.10 or 0.15 or 0.20 or 0.30 with respect to the hourly value of the corresponding standard hour. It can be seen that high values of probability of a variability greater than $10 \%$ are found around sunrise and also at sunset during winter and equinoxes, otherwise the variability can be assumed to be an «intrinsic noise» during all the other hours of the day.

Figure 2 shows the probability the variability within-the-hour, positive or negative, be greater than a given level at different seasons. The data used are those measured at Matera during the period from 1993 to 1999 . The difference with season, regarding the time of appearance of high probabilities and the value of probability is evident. Clearly, the variability is rather negligible during day-time hours.

Figure 3 illustrates the upper and lower deciles of the variability in TEC from hourly measurements and from 10-min measurements made at Matera and Hailsham. They are deter- mined for each of the two locations from the respective relative deviations counted according to eq. (2.1) and (2.3) and from all the avaliable data measured at each one location, respectively. Figure 3 clearly shows the difference between bounds of variability from day-to-day and those of the within-the-hour variability. In this last case the variability is limited within a range of values less than \pm 0.05 except at sunrise and also at sunset in winter or during local disturbed conditions (see also tables I and II). It can be stated that the present analysis shows clearly that the within-the-hour variability in TEC is very low, practically negligible, except at sunrise and sunset. We may therefore assume that TEC is stationary in the interval time of one hour contrary to $f o F 2$, which seems to have a stationarity of less than $10 \mathrm{~min}$.

Variability in foF 2 - The analysis points out that the within-the-hour variability in $f o F 2$ may reach values greater, in absolute value, than $20 \%$ 

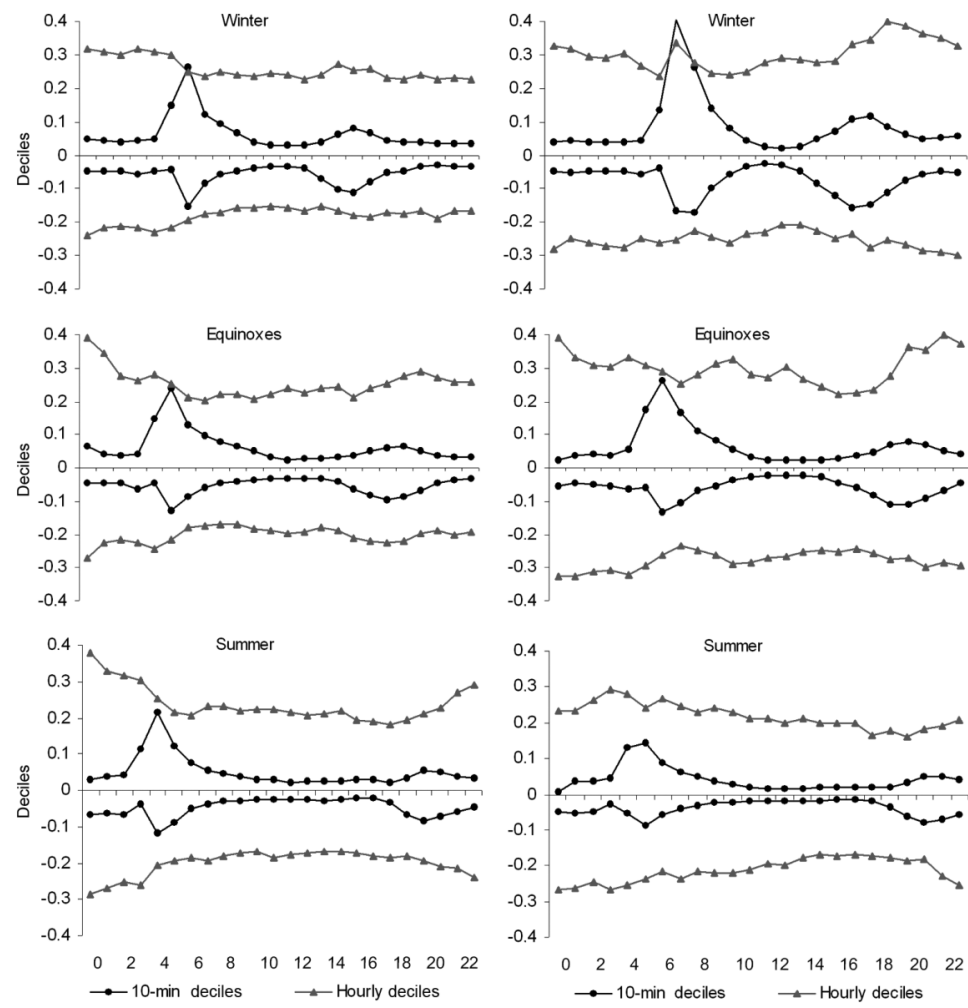

Fig. 3. Deciles of TEC from hourly daily values and deciles from TEC measured every 10-min at Matera (left) and Hailsham (right).

Table IV. Probability the variability within-the-hour in $f o F 2$ be greater than 0.10 (A), 0.15 (B), 020 (C). Data from Rome measured during 1997 (November, December), 1999 (June, July, August, September), 2001 (January, February, March, April). Black lines are due to missing measurements in May and October.

\begin{tabular}{c|ccccccccccccccccccccccccc}
\hline \hline $\mathbf{A}$ & 0 & 1 & 2 & 3 & 4 & 5 & 6 & 7 & 8 & 9 & 10 & 11 & 12 & 13 & 14 & 15 & 16 & 17 & 18 & 19 & 20 & 21 & 22 & 23 \\
\hline $\mathbf{J}$ & 9.8 & 9.4 & 9.1 & 20 & 23 & 20 & 68 & 39 & 4.6 & 24 & 16 & 16 & 16 & 12 & 12 & 12 & 35 & 35 & 24 & 24 & 33 & 17 & 22 & 12 \\
$\mathrm{~F}$ & 8.1 & 17 & 16 & 14 & 14 & 17 & 68 & 19 & 5.6 & 5.9 & 11 & 19 & 15 & 18 & 6.9 & 8.7 & 16 & 17 & 8.1 & 23 & 24 & 25 & 24 & 18 \\
$\mathrm{M}$ & 13 & 11 & 12 & 12 & 11 & 37 & 30 & 7 & 8.6 & 12 & 14 & 15 & 29 & 13 & 15 & 6.6 & 12 & 15 & 27 & 12 & 20 & 22 & 16 & 16 \\
$\mathrm{~A}$ & 14 & 11 & 15 & 7.7 & 10 & 14 & 7 & 8.4 & 11 & 14 & 11 & 12 & 9.2 & 11 & 11 & 2.4 & 7.6 & 10 & 27 & 17 & 12 & 13 & 16 & 17 \\
$\mathbf{M}$ & & & & & & & & & & & & & & & & & & & & & & & & \\
$\mathrm{J}$ & 24 & 13 & 12 & 14 & 7.8 & 10 & 6 & 6.4 & 7.3 & 14 & 12 & 13 & 13 & 6.8 & 8.5 & 9.6 & 8.8 & 4.4 & 7.6 & 18 & 20 & 16 & 25 & 21 \\
$\mathrm{~J}$ & 16 & 11 & 13 & 8.2 & 8.8 & 9.7 & 13 & 9.9 & 12 & 8.8 & 5.2 & 6.1 & 8.7 & 6.5 & 8 & 9.6 & 8.4 & 13 & 7 & 18 & 19 & 13 & 15 & 12 \\
$\mathrm{~A}$ & 26 & 9.6 & 22 & 20 & 12 & 22 & 11 & 10 & 10 & 7.3 & 6.6 & 9.1 & 9.1 & 1.9 & 4.8 & 1.3 & 0.6 & 6.2 & 6.7 & 21 & 20 & 34 & 20 & 14 \\
$\mathrm{~S}$ & 13 & 19 & 18 & 9.8 & 8 & 39 & 18 & 13 & 5.7 & 4.4 & 2.2 & 1.3 & 5.5 & 6.6 & 4.9 & 1.6 & 7.1 & 14 & 9.4 & 11 & 15 & 15 & 13 & 14 \\
$\mathrm{O}$ & & & & & & & & & & & & & & & & & & & & & & & & \\
$\mathrm{N}$ & 13 & 10 & 16 & 20 & 28 & 22 & 71 & 16 & 11 & 9.5 & 9.5 & 15 & 17 & 8.2 & 15 & 27 & 58 & 39 & 24 & 26 & 21 & 20 & 2.9 & 7.2 \\
$\mathrm{D}$ & 6.4 & 10 & 3.5 & 16 & 25 & 32 & 70 & 26 & 28 & 24 & 17 & 19 & 18 & 20 & 21 & 24 & 42 & 29 & 23 & 7.7 & 11 & 20 & 15 & 15 \\
\hline
\end{tabular}


Table IV (continued).

\begin{tabular}{|c|c|c|c|c|c|c|c|c|c|c|c|c|c|c|c|c|c|c|c|c|c|c|c|c|}
\hline & 0 & 1 & 2 & 3 & 4 & 5 & 6 & 7 & 8 & 9 & 10 & 11 & 12 & 13 & 14 & 15 & 16 & 17 & 18 & 19 & 20 & 21 & 22 & 23 \\
\hline & 8 & & & 10 & 6 & 9.1 & 48 & 0 & 0.7 & 6 & 7.3 & 3 & 8 & 3.7 & 0 & 2 & 19 & 24 & 12 & 11 & 14 & 6 & 4 & . \\
\hline $\mathrm{F}$ & .7 & 9.1 & 9.2 & 9.4 & 4.2 & 9.2 & 49 & 4.9 & 0.4 & 1.5 & 4.5 & 8.6 & 1.6 & 5 & 1.7 & 5.2 & 6.6 & 1 & 1 & 6.6 & 13 & 3 & 12 & 9.6 \\
\hline M & 6.5 & 3 & 7.6 & 5.3 & 5 & 22 & 13 & 9 & 3.2 & 3.5 & 4 & 2 & 11 & 2 & 6.6 & 0.3 & 5.3 & 7 & .7 & 7.3 & 9 & 11 & 9.4 & 8.8 \\
\hline A & 11 & 9.2 & 12 & 5 & 7.1 & 6.1 & 2.9 & 3 & 4.3 & 6.2 & 3.4 & & 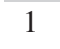 & & 6 & 0.9 & 0.3 & 9 & 7 & 7 & 1 & 6.1 & 15 & 12 \\
\hline & & & & & & & & & & & & & & & & & & & & & & & & \\
\hline J & 7 & 11 & 7.3 & 1 & 2.9 & 6.5 & 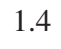 & 0.8 & 0.8 & 3.8 & 3.8 & 2 & 3.2 & 1 & 4.8 & 5 & 7.7 & & 3 & 16 & 4 & 0 & 19 & 13 \\
\hline 1 & 12 & 9.2 & 6.8 & 3.43 & 3.4 & 3.8 & 9.7 & 7.4 & 3.1 & 4.4 & 2.6 & 0 & 6 & 2 & 3.8 & 2.6 & 4.8 & & 4 & 5 & 17 & 10 & 9.7 & 9 \\
\hline A & 22 & 8 & 17 & 15 & 5 & 7.6 & 5 & 6 & 3 & 3.7 & 2.6 & 2.9 & 2.8 & & 0.7 & 0.3 & 0 & & 2 & 6 & 14 & 2 & 14 & 9.4 \\
\hline S & 7.2 & 15 & 12 & 4.43 & 3.5 & 22 & 2.3 & 1.5 & 2.7 & 2.6 & 0.4 & 0.4 & 0.7 & 5 & 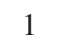 & 0 & 1.3 & .7 & 5 & 5.3 & 7 & 3.8 & 8.3 & 7.1 \\
\hline$N$ & & 3. & 11 & 9. & 8.3 & 8.4 & 47 & 5.5 & 2 & 3.6 & 1.5 & & 4. & 0 & 1 & 7.2 & 36 & & 2 & 11 & 8.1 & 12 & .4 & 4.1 \\
\hline D & 0 & 6 & 1.8 & 5.8 & 11 & 17 & 52 & 11 & 12 & 12 & 5.8 & 7.5 & 8.7 & 6.6 & 12 & 9 & 27 & 14 & 16 & 3.8 & 2.7 & 8.8 & 8.9 & 8.3 \\
\hline 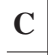 & 0 & 1 & 2 & 3 & 4 & 5 & 6 & 7 & 8 & 9 & 10 & 11 & 12 & 13 & 14 & 15 & 16 & & & 19 & 20 & 1 & 22 & 23 \\
\hline J & & 3.2 & 0 & . & 3 & 1.0 & & 0 & & & 1 & & 3.8 & & 0.1 & & 12 & & & & & 2 & & \\
\hline $\mathrm{F}$ & 1.9 & 6 & 4.9 & 5.5 & 1.6 & 3.6 & 30 & 1.5 & 0 & 0 & 1.2 & 1.6 & 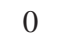 & 2 & 0 & 3.6 & 4.5 & 3.6 & 0.4 & 1.6 & 5.7 & 8.8 & 5.6 & 6.2 \\
\hline M & 5.9 & 5.3 & 4.7 & 2.6 & 2.6 & 11 & 4.4 & 0.6 & 1.6 & 1.7 & 1.8 & 1.4 & 3.8 & 0.4 & 4.6 & 0 & 3.2 & 2.4 & 2.7 & 3.5 & 4.4 & 7.6 & 5 & \\
\hline A & 10 & 7.3 & 9.7 & 3.1 & 6.2 & 2.8 & 1.3 & 2 & 1.4 & 2.4 & 1.6 & 1.3 & 0.3 & ). 6 & 0.6 & 0 & 0.3 & 0 & 5.5 & 3.6 & 5.5 & 3.7 & 13 & 5.2 \\
\hline & & & & & & & & & & & & & & & & & & & & & & & & \\
\hline $\mathrm{J}$ & 9.8 & 9 & 4 & 8.9 & 2 & 3.9 & 1. & 0 & 0 & 1 & 1.4 & 0.4 & 0.7 & 0 & 6 & 3.7 & 6.4 & & 2 & 15 & 13 & 5.9 & 13 & 10 \\
\hline $\mathrm{J}$ & 9.2 & 7.2 & 2.1 & 2.4 & 1 & 3.1 & 7.4 & 5.4 & 0.5 & 1.3 & 1.5 & 0 & 4.1 & 0.5 & 1.3 & 1.7 & 1.3 & $\varepsilon$ & 3.7 & 13 & 13 & 7.5 & 9 & 5.8 \\
\hline A & 15 & 5.6 & 13 & 12 & 2.4 & 3.4 & 3.8 & 3.3 & 1.5 & 2 & 0.4 & 0.4 & 0.4 & 4 & 0.3 & 0.3 & 0 & 2 & 1.3 & 13 & 9.6 & 14 & 11 & 7.9 \\
\hline S & 4.8 & 11 & 6.6 & 3.7 & 1.7 & 9.7 & 0 & 0.7 & 0.4 & 0.4 & 0 & 0 & 0.4 & 3.8 & 0 & 0 & 0 & y & 1 & .7 & 0.6 & 1 & .3 & 3.4 \\
\hline & & & & & & & & & & & & & & & & & & & & & & & & \\
\hline $\mathrm{N}$ & 3.6 & 0.9 & 4.5 & 1 & 0.9 & 3.2 & 26 & 2.3 & 0 & & 0. & & & & & 2.2 & 25 & & 2 & 1.7 & & & 0 & 1 \\
\hline & 0 & 0.6 & 0 & 3.5 & 5.7 & 7.6 & 37 & 4 & 8.6 & 4.7 & 1.7 & 0.5 & 6.7 & 2.7 & 2.2 & 3.4 & 13 & 4.1 & 6.7 & 0.6 & 1.8 & 3.5 & 8.1 & 5.1 \\
\hline
\end{tabular}

and less than around $30 \%$ of the corresponding hourly value measured at the standard hour during disturbed conditions or around sunrise and sunset (table IV). It is also clear that a withinthe-hour variability around $12 \%$, positive or negative, is always present throughout the day.

Figure $4 a, b$ reports the curves of probabilities which show that the variability within-thehour could exceed a given value. It can be seen that the probability of high values of variability exist during winter and equinoxes. They are more frequent from sunset up to sunrise and seem to be related to disturbed conditions $(\mathrm{Bu}-$ resova and Laštovička, 2001). On the other hand, the probability of variability values greater than 0.30 (in absolute value) of the corresponding hourly value is very small except at summer and usually after sunset up to midnight. Figure 5 illustrates the probabilities in each season that the variability in $f o F 2$ exceeds a given value. This figure confirms the above mentioned statements. It is evident that the variability within-the-hour in foF 2 is bounded between a positive and a negative level of 0.10 to 0.15 . Values of variability greater than 0.30 may appear mostly in summer after sunset, but the probability is small, less than $10 \%$.

Figure 6 shows the upper and lower deciles of variability in foF 2 from hourly measurements and from 5-min made in Rome. They are calcu- 
(a)
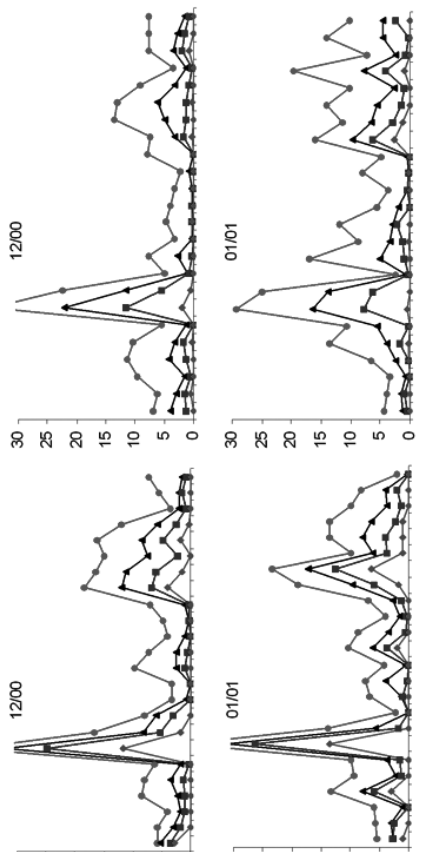

이뮤요으 응

(\%) Ky!lqeqojd

(๘)
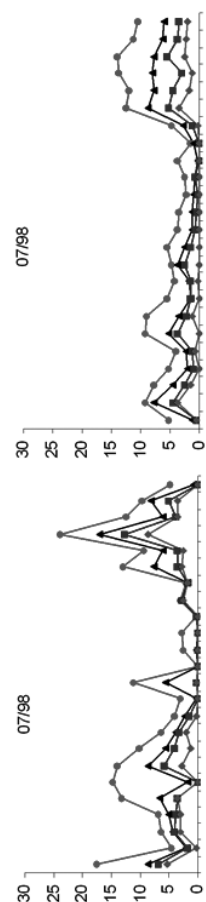

(\%) Kyllqueqoid

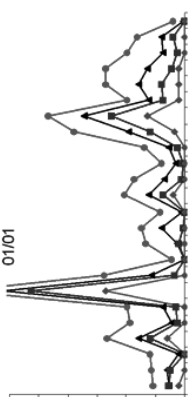

(\%) Kingeqold

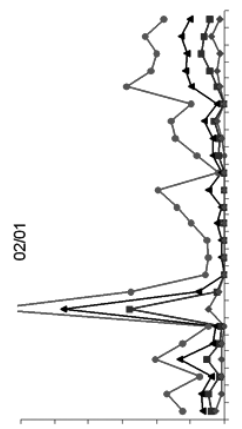

ำ ㄴำ 으

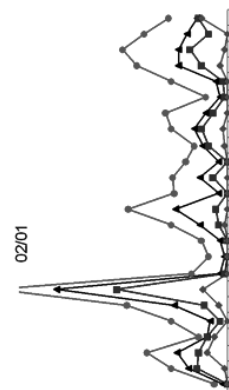

요 으 느 으 ᄂ

(\%) Kungeqoud

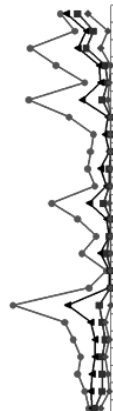

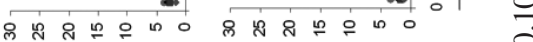

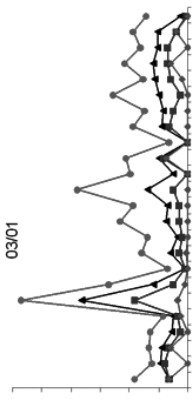

노 조 느으

(\%) Kyllqeqodd
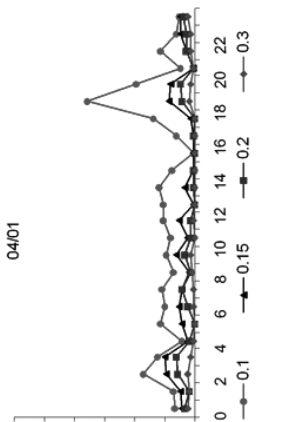

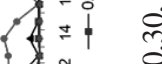

$\left\{\begin{array}{lll}1 & =1\end{array}\right.$

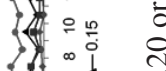

III + i

It 0 ?

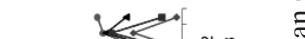

นิ

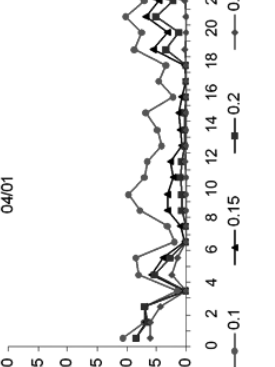

(\%) Kryqueqoid
If

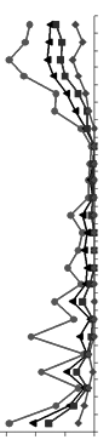

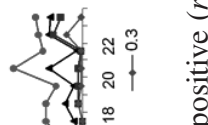

$\exists$

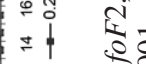

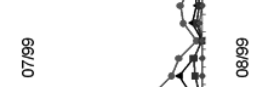

要

$\frac{8}{8}$
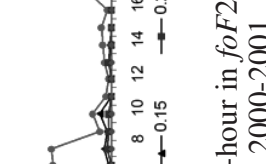

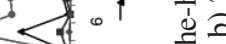

A 4 क

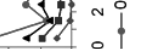

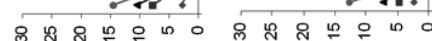

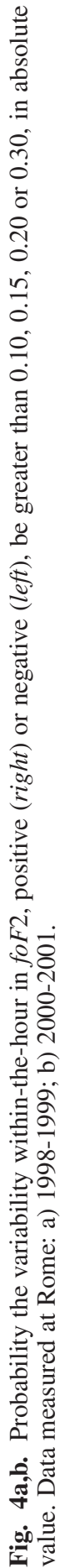

น

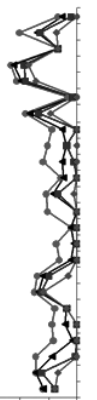

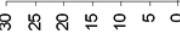

(\%) Kinlqueqoud
(\%) 세llqeqodd

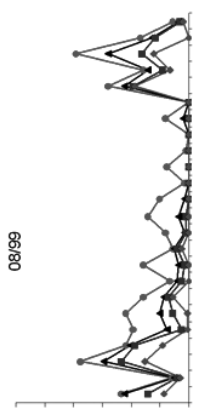

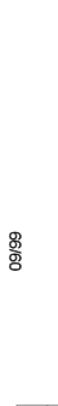

은 2 은

(\%) Kupqeqoud

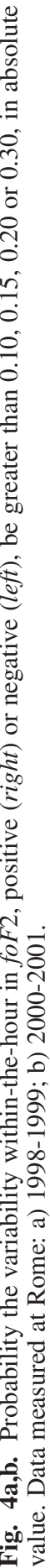



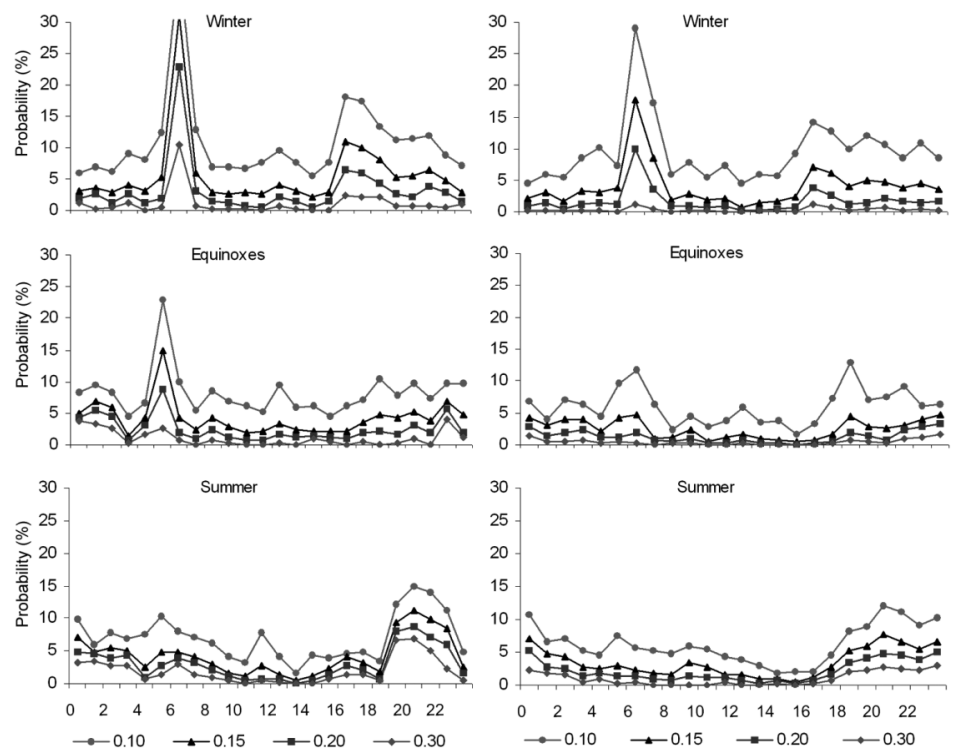

Fig. 5. Probability the variability within-the-hour in $f_{o} F 2$, positive (right) or negative (left), be greater than $0.10,0.15,0.20$ or 0.30 , in absolute value, using all the available data from Rome.
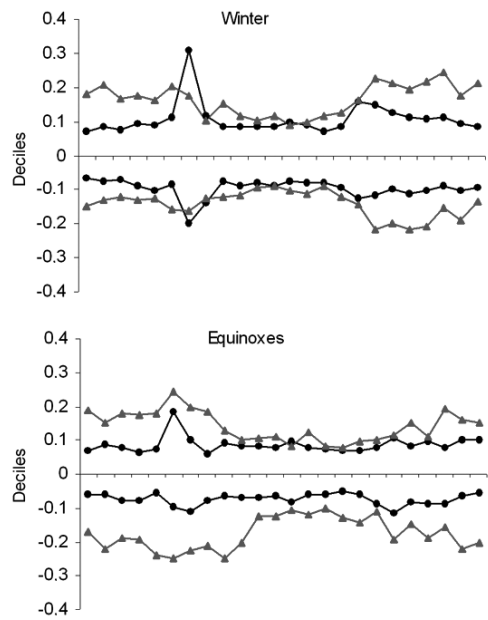

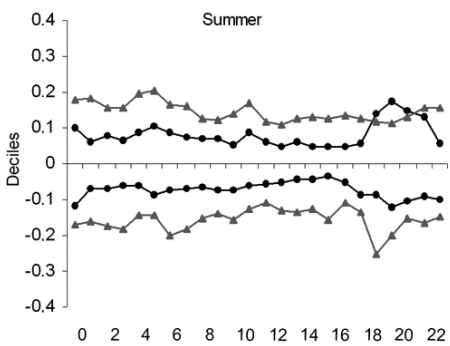

$\rightarrow$ - - min deciles $\quad \rightarrow$ Hourly deciles

Fig. 6. Deciles of $f o F 2$ from hourly daily values and deciles from foF 2 5-min measurements made in Rome during 1997-2001.

lated from all the available measurements using eq. (2.2) and a similar one for the hourly deciles (Fotiadis et al., 2001). Figure 6 shows clearly the existing difference between day-to-day and with- in-the-hour variability. The bounds of variability for the $90 \%$ of the time are around $20 \%$ in absolute value in the case of the day-to-day variability (Kouris and Fotiadis, 2002) whereas for 
the within-the-hour these bounds are around $10 \%$ in absolute value with respect to the standard hour value. Exceptions occur again at sunrise in winter and equinoxes, and around sunset in summer (fig. 6) where these limits are more pronounced.

Variability and disturbances - Buresova and Laštovička (2001) selecting strong geomagnetic storms found that during ionospheric disturbances there is a considerable daytime within-the-hour variability in $f o F 2$. The results reported here confirm the strong variability. Indeed, although table IV refers to monthly conditions, it clearly shows that a substantial variability exists throughout the day, being more pronounced around sunrise and sunset. On the contrary, the within-the-hour variability in TEC is around $5 \%$ of the corresponding hourly daily value except around sunrise and sunset, and during local disturbed conditions where values of the order of $20 \%-30 \%$ are reached. However, it is known that ionospheric disturbances may follow magnetic disturbances (fig. 7) but there is no simple direct relationship between them. Indeed, we have observed that when $K p$ is much greater than 2 the level of the within-thehour variability in foF 2 could be that of the «quiet variability» (fig. 8), that is about 0.12 or less, which is of the same order of the variations observed in the critical frequency of the E-layer (Kouris and Fotiadis, 2002). On the other hand, the variability in TEC is around $5 \%$ or less, except at sunrise (figs. 7 and 8).

Figure 9 shows clearly that although the $K p$ index is less than 2, the variability in $f o F 2$ is quite substantial, reaching values around 0.20; whereas that in TEC is negligible apart from around sunrise. We may therefore state that there is nearly always a substantial variability in foF 2 in the interval time of an hour, whereas the with-
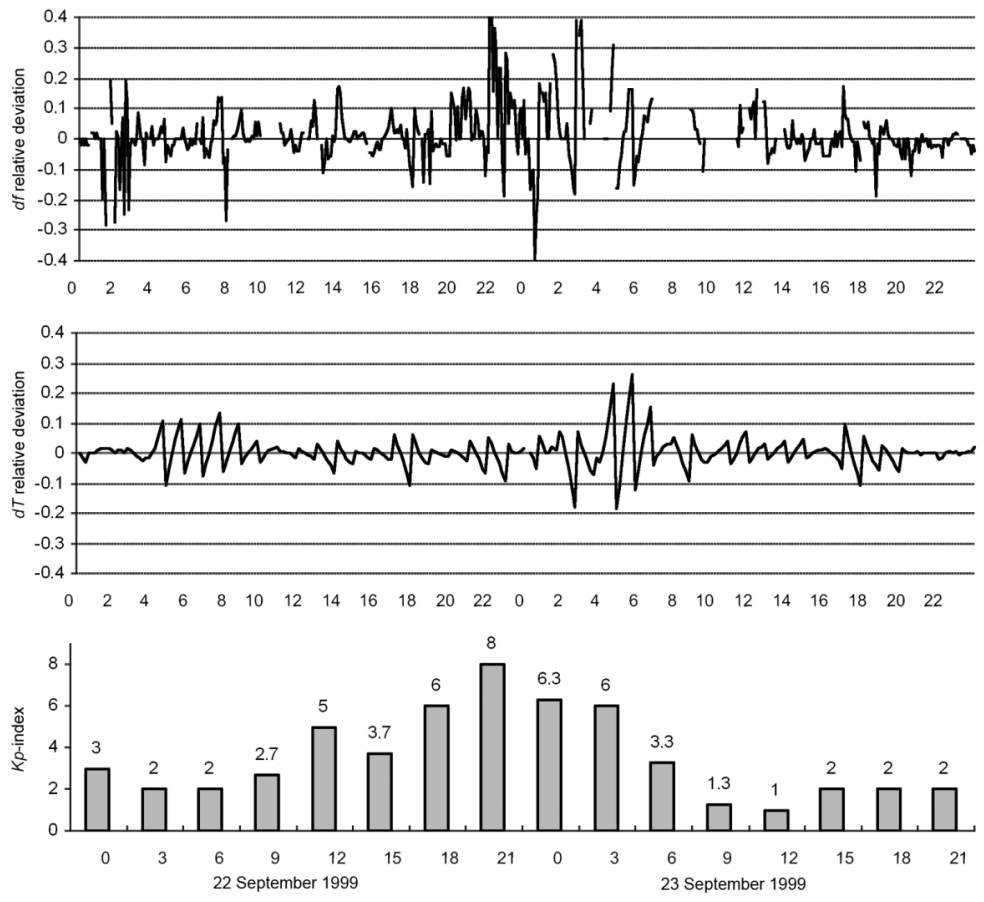

Fig. 7. Relative deviations of 5-min foF2 measurements (Rome, 22-23 September 1999) and 10-min relative deviations of TEC measurements (Matera, 22-23 September 1999). Values of $K p$ index. 

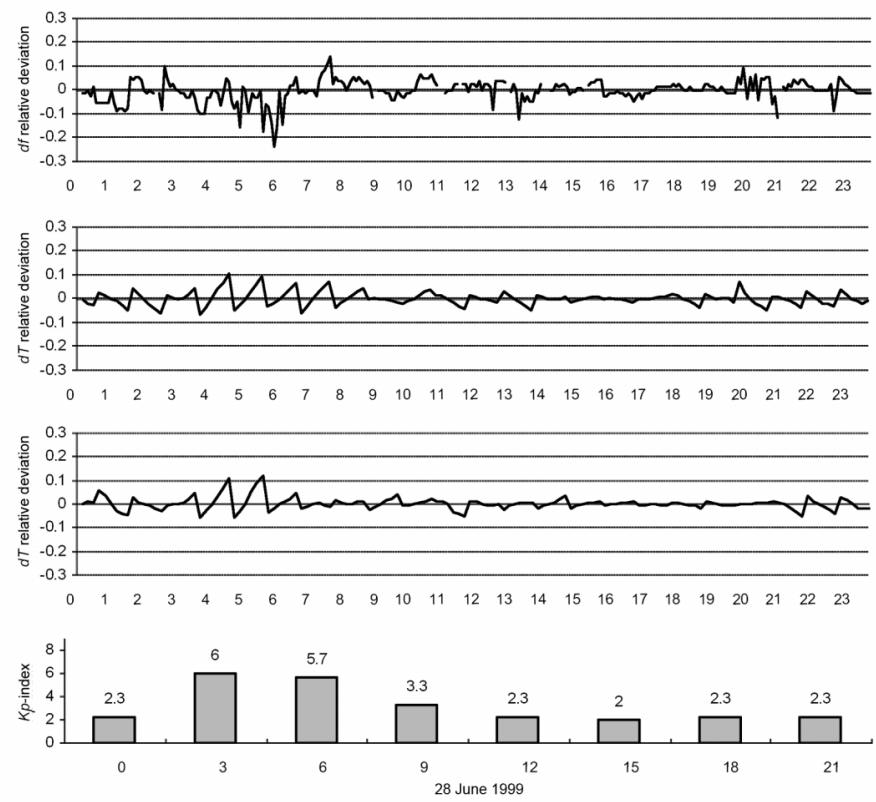

Fig. 8. Relative deviations of $f o F 2$ from measurements made in Rome (upper) and of TEC made at Matera (upper middle) and Hailsham (low middle); values of $K p$ index (bottom).
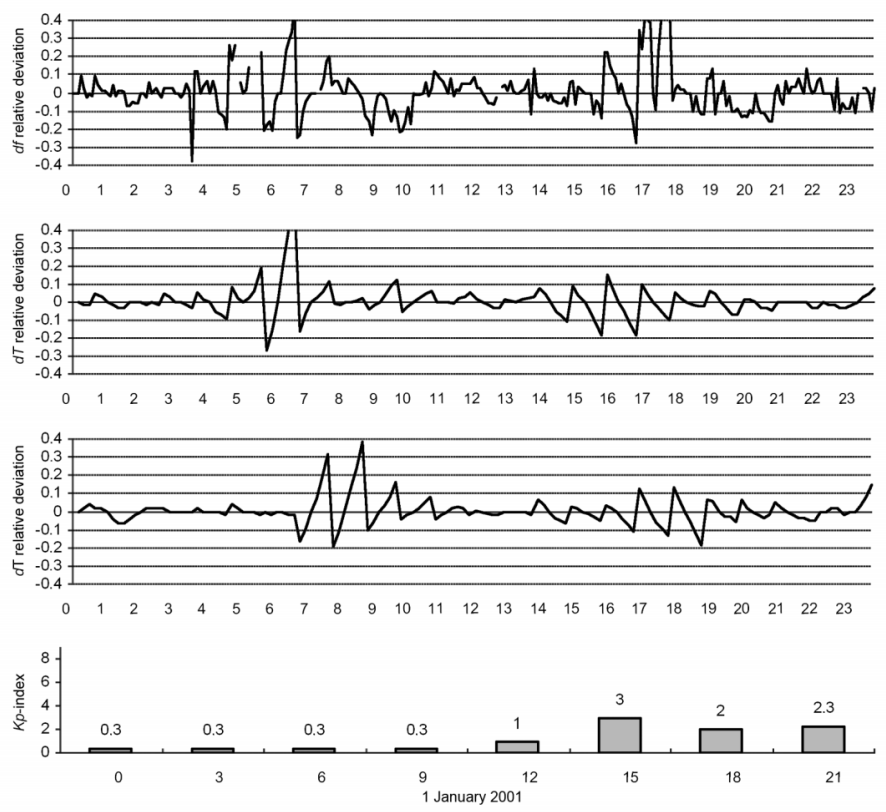

Fig. 9. Relative deviations of 5-min $f o F 2$ measurements from Rome (upper) and 10-min TEC measurements from Matera (upper middle) and Hailsham (low middle); values of $K p$ index (bottom). 
in-the-hour variability in TEC is about $5 \%$ or less apart from during local disturbed conditions (table II) and at sunrise and sunset. In these last cases the pronounced variations in TEC are simply due to its rapid increase or decrease during its diurnal normal variation, that is at sunrise and sunset when rapid changes in the atmospheric conditions occur. The present study also points out that ionospheric disturbed conditions are more marked in $f o F 2$ rather than in TEC and this is in agreement with previous findings regarding other ionospheric parameters (Kouris and Fotiadis, 2002).

\section{Conclusions}

The obtained results lead to the conclusion that the deciles of the within-the-hour variability in foF 2 are $12 \%$ higher/lower than the corresponding standard hourly value of $f_{o} F 2$, at least regarding the region of Rome. Variations higher, that is of the order of $20 \%-30 \%$, are usually observed at sunrise and sunset due to changes in the atmospheric conditions. Moreover, large variations (positive/negative) in the interval time of one hour of the same order ( 0.20 to 0.30$)$ due to disturbed conditions are also observed, but their probability of appearance is less than $5 \%$ per month.

The variations in TEC for $90 \%$ of the time are usually less than $\pm 5 \%$ of the corresponding hourly value. However, at sunrise and also at sunset in winter variations of the order of $20 \%$ $30 \%$ are observed and should be attributed to the rapid increase/decrease of TEC during its diurnal normal variation. They depend strongly on season. The analysis shows also that a stationarity in TEC of about one hour may be accepted.

In addition ionospheric disturbances affect the $F 2$ layer maximum electron density rather than TEC in the interval time of one hour.

\section{Acknowledgements}

This work is part of a joint Greek-Italian research project supported by the cultural collaboration (7th protocol) between the two countries.

\section{REFERENCES}

Buresova, D. and J. LAŠTOVIČKA (2001): Changes in the $F 1$ region electron density during geomagnetic storms at low solar activity, J. Atmos. Solar-Terr. Phys., 63, 537-544.

Fotiadis, D.N., S.S. KouRIS and B. Zolesi (2001): Preliminary results on the within-the-hour ionospheric variability, Phys. Chem. Earth (C), 26 (5), 315-318.

Fotiadis, D.N., S.S. Kouris, V. Romano and B. Zolesi (2004): Climatology of ionospheric $F$-region disturbances, Ann. Geophysics, 47 (4), 1311-1323.

KouRIS, S.S. and D.N. FotIADIS (2002): Ionospheric variability: a comparative statistical study, Adv. Space Res. 29 (6), 977-985.

KouRIS, S.S., D.N. Fotiadis and T.D. Xenos (1998): On the day-to-day variation of foF2 and $M(3000) F 2, A d v$. Space Res., 22 (6), 872-876.

KouRIS, S.S., D.N. Fotiadis and B. Zolesi (1999): Specifications of the $F$-region variations for quiet and disturbed conditions, Phys. Chem. Earth (C), 24 (4), 321-327.

Kouris, S.S., B. Zolesi, D.N. Fotiadis and C. Bianchi (2000): On the variability within-the-hour and from hour-to-hour of the $F$-region characteristics above Rome, Phys. Chem. Earth (C), 25 (4), 347-351.

Kouris, S.S., K.V. Polimeris and LJ.R. CANDER (2006): Specifications of TEC variability, Adv. Space Res., 37 (5), 983-1004.

Polimeris, K.V., V. Romano, S.S. Kouris and B. Zolesi (2004): The within-the-hour variability in TEC: some preliminary results, Bulg. Geophys. J., 30 (1-4).

RAwER, K. (1957): The Ionosphere (F. Ungar, New York).

Rawer, K. (1993): Wave Propagation in the Ionosphere, (Kluwer Publ. Co., Dordrecht NL).

RAwER, K., S.S. Kouris and D.N. Fotiadis (2003): Variability of $F 2$ parameters depending on modip, $A d v$. Space Res., 31 (3), 537-541.

Zolesi, B., S.S. Kouris, D.N. Fotiadis and C. Scotto (2001): Evaluation of the ionospheric noise and variability within-the-hour by $5 \mathrm{~min}$ ionospheric soundings, Phys. Chem. Earth (C), 26 (5), 359-362.

(received October 19, 2005; accepted June 20, 2006) 